Article Title

\title{
Risk of Severe COVID-19 Outcomes Among Patients with Rheumatoid Arthritis in the United States
}

\section{Corresponding Author}

Full Name

Vincent Yau

Postal Address

Email

\author{
Genentech \\ Building 40 \\ 600 E Grand Ave \\ South San Francisco, 94080 \\ yau.vincent@gene.com
}

\section{Co-Authors}

Full Name

Ching-Yi Chuo

Vince Yau

Sriraman Madhavan

Larry Tsai

Jenny Chia
Department

Institution

City

Country

Department

Institution

City

Country

Department

Institution

City

Country

Department

Institution

City

Country

Department

Institution

City

Country
Personalized Health Care

Genentech

South San Francisco

United States

Personalized Health Care

Genentech

South San Francisco

United States

Personalized Health Care

Genentech

South San Francisco

United States

Product Development

Genentech

South San Francisco

United States

Personalized Health Care

Genentech

South San Francisco

United States 
medRxiv preprint doi: https://doi.org/10.1101/2021.07.09.21260106; this version posted July 12, 2021. The copyright holder for this preprint

(which was not certified by peer review) is the author/funder, who has granted medRxiv a license to display the preprint in perpetuity.

All rights reserved. No reuse allowed without permission.

Competing Interests: All authors on this paper are employed by Genentech, which is a member of the Roche Group.

Contributorship: All authors in this manuscript contributed in a meaningful and significant manner.

\section{Acknowledgements: N/A}

Funding Info: This study was funded by Genentech.

\section{Ethical approval information:}

The protocol for this study is in line with the Declaration of Hensinki (2008). The authors conducted secondary research using de-identified data licensed from TriNetX in compliance with 45 CFR 164.514(a)-(c). The data had identifying information removed and was coded in such a way that the data could not be linked back to subjects from whom it was originally collected.

This research utilized the data licensed by TriNetX and described above, does not require IRB or ethics review, as analyses with this data do not meet the definition of "research involving human subjects" as defined within 45 CFR 46.102(f) which stipulates human subjects as living individuals about whom an investigator obtains identifiable private information for research purposes. Neither the provider of the data nor the researchers were able to link the data with identifiable individuals. Consent to participate was not applicable in this retrospective observational study of de-identified data.

Data Sharing Statement: Data may be obtained from a third party and are not publicly available. This study used data provided by the TriNetX (Cambridge, MA) DataWorks network, which includes 41 Healthcare Organizations (HCOs) and 1,016,769 patients across the United States as of 20 August 2020. In order to use these data, Genentech purchased a license from TriNetX.

Patient and Public Involvement: We included the following statement in the main manuscript: "While this study used patient level data from a large electronic medical record database, no patients or members of the public contributed to the design or analysis of this study."

\section{Key Messages:}

- $\quad$ Patients with Rheumatoid Arthritis (RA) tend to be older, and often have co-morbidities which could put them at greater risk of severe COVID-19 outcomes.

- This study is one of the largest studies of COVID-19 infected RA populations to date. We did not find increased risk of hospitalization, ICU admission, or mortality among RA patients vs. matched non-RA patients.

- Patients previously exposed to anti-coagulants experienced higher risks of hospitalization and overall mortality. Extra attention is needed for treating such patients. 
medRxiv preprint doi: https://doi.org/10.1101/2021.07.09.21260106; this version posted July 12, 2021. The copyright holder for this preprint (which was not certified by peer review) is the author/funder, who has granted medRxiv a license to display the preprint in perpetuity.

All rights reserved. No reuse allowed without permission.

\title{
Abstract \\ Introduction
}

Coronavirus disease 2019 (COVID-19) has infected over 22 million individuals worldwide. It remains unclear whether patients with COVID-19 and Rheumatoid Arthritis (RA) experience worse clinical outcomes compared to similar patients with COVID-19 without RA.

\begin{abstract}
Aim
The aim of this study is to provide insights on how COVID-19 impacted patients with RA given the nature of the disease and medication used.

\section{Methods}

RA cases were identified via International Classification of Diseases (ICD) codes and COVID-19 cases by laboratory results in the U.S. based TriNetX network. Patients with COVID-19 and RA were propensity-score matched based on demographics with patients with COVID-19 without RA at a 1:3 ratio. A hospitalized sub-population was defined by procedure codes.
\end{abstract}

\section{Results}

We identified 1,014 COVID-19 patients with RA and 3,042 non-RA matches selected from 137,757 patients. The odds of hospitalization (non-RA:23\%, RA:24.6\%, OR:1.08, 95\% Cl: 0.88 to 1.33 ) or mortality (non-RA:5.4\%, RA:6\%, OR:0.93, 95\% Cl: 0.65 to 1.34 ) were not significantly different.

The hospitalized sub-population included 249 patients with COVID-19 and RA and 745 non-RA matches selected from 21,435 patients. The risk of intensive care unit (ICU) admission (non$\mathrm{RA}: 18.8 \%, \mathrm{RA}: 18.1 \%$, OR:0.94, 95\% Cl: 0.60 to 1.45), and inpatient mortality (non-RA:14.4\%, RA: $14.5 \%$, OR:0.86, $95 \%$ Cl: 0.53 to 1.40 ) were not significantly different.

\section{Conclusion}

We didn't find evidence suggesting patients with COVID-19 and RA are more likely to have severe outcomes than patients with COVID-19 without RA.

\section{Keywords}

Rheumatoid Arthritis; COVID-19; hospitalization; mortality; intensive care unit. 
medRxiv preprint doi: https://doi.org/10.1101/2021.07.09.21260106; this version posted July $12,2021$. The copyright holder for this preprint (which was not certified by peer review) is the author/funder, who has granted medRxiv a license to display the preprint in perpetuity.

All rights reserved. No reuse allowed without permission.

\section{Introduction}

Coronavirus disease 2019 (COVID-19) has, as of 20 August 2020, infected over 222 million individuals worldwide and caused over 783,000 deaths. ${ }^{1}$ Age is the most important risk factor for mortality. ${ }^{2}$ Older individuals account for $80 \%$ of hospital admissions for COVID-19 infection and mortality risk among hospitalized individuals over 65 years old is 23 times greater than individuals under $65 .^{3}$

Increased age is also associated with increased prevalence of comorbidities such as diabetes and hypertension which could contribute to worse outcomes in COVID-19 infected individuals. ${ }^{4}$ While several comorbidities such as obesity have been associated with worse outcomes, ${ }^{5}$ it is still unclear whether or not patients with COVID-19 and Rheumatoid Arthritis (RA) experience worse clinical outcomes compared to COVID-19 patients without RA. Patients with RA have higher risk of infections due to disease-related immune dysregulation and RA treatments which

modulate patient immune responses. ${ }^{67}$ This has raised patient concerns about whether or not to continue RA treatments during the pandemic. ${ }^{8}$

Ironically, several RA medications, rather than being seen as risk factors, have been evaluated as COVID-19 treatments. Several immune modulating RA drugs like dexamethasone have seen widespread use around the world for COVID-19, and others are being evaluated such as interleukin6 (IL-6) inhibitors due to hypotheses that they might reduce risk of severe immune mediated COVID-19 outcomes. ${ }^{9}$

In this study, we compare the health outcomes COVID-19 positive patients with and without RA. This analysis will provide insight into how COVID-19 has affected this vulnerable population.

\section{Methods \\ Data Source}

The study used the TriNetX (Cambridge, MA) DataWorks network with 41 Healthcare Organizations (HCOs) and 1,016,769 patients across the United States. This COVID-19 specific dataset includes electronic medical records (diagnoses, procedures, medications, and laboratory values) primarily from large academic institutions with better representation of the south $(41 \%$, west $(23 \%)$, midwest (15\%) and northwest $(17 \%))$.

\section{Study Population}

Patients were required to have confirmed COVID-19 as defined by meeting one of three criteria: 1) Patients with at least one occurrence of International Classification of Diseases, 10th Revision, Clinical Modification (ICD10-CM) code U07.1 or 2) at least one occurrence of ICD10-CM code B97.29 without a negative COVID-19 laboratory test within 7 days or 3) positive antibody or RNA based COVID-19 laboratory test. The study period was between 20 February and 20 August 2020. Index date was defined as the first date fulfilling any of the above definitions.

Patients with COVID-19 were then classified as with RA or without RA. RA was defined as having at least two occurrences of ICD9-CM code 714.0 or corresponding ICD10-CM codes (online supplemental table S1) anytime in their medical records. We also collected information on all RA specific and other treatments such as Disease-Modifying Antirheumatic Drugs (DMARD) such as conventional synthetic (CS), targeted synthetic (TS), and Biologics ([B], 
medRxiv preprint doi: https://doi.org/10.1101/2021.07.09.21260106; this version posted July 12, 2021. The copyright holder for this preprint (which was not certified by peer review) is the author/funder, who has granted medRxiv a license to display the preprint in perpetuity.

All rights reserved. No reuse allowed without permission.

steroids, anti-coagulants and antibiotics; online supplemental table S2) taken within the year prior to index date. There are two main populations: 1) individuals who met our COVID-19 definitions and 2) a sub-population of hospitalized patients as defined by procedure codes (table S3).

\section{Outcomes}

The outcomes of interest for all COVID-19 patients were hospitalization and post COVID-19 mortality reported by the HCOs. For the hospitalized sub-population, the outcomes of interest were ICU admission and mortality (online supplemental table S3).

\section{Statistical Analysis}

For each patient-population, we conducted 1:3 propensity score matching using the nearest neighbor method with 0.1 caliper for age, gender, race, and smoking status on their RA status. Two-proportion-z-tests were used to compare the crude proportions of outcomes. Multiple logistic regressions controlling for matching variables, Charlson Comorbidity Index $(\mathrm{CCl})$, Body Mass Index (BMI), and prior medication were used to derive outcome estimates. All analyses were conducted using $\mathrm{R}(3.5 .1)$

\section{Sensitivity Analysis}

Sensitivity analyses were conducted by restricting the COVID-19 definition to only lab confirmed cases.

\section{Ethics}

This study used de-identified patient level data from a large electronic medical record database that met Section 164.514 (a) of the HIPAA privacy rule and therefore not considered "research involving human subjects".

\section{Results}

We identified 138,792 COVID-19 cases and among them, 1,035 were RA patients. Patients with RA and COVID-19 were older (without RA, with RA median age: 44, 63), more likely to be female (without RA, with RA: $55 \%, 79 \%$ ), and non-Hispanic (see Table 1). RA patients were also more likely to be smokers (without RA, with RA: $6.5 \%, 24.5 \%$ ), with slightly higher BMI and comorbidity burden. RA patients had higher prior-year medication utilization across all treatment classes. However, since the utilization of tsDMARDs was low $(n=93)$, we did not separately assess tsDAMRD use.

Comparing the pre-matched crude proportions of the outcomes between COVID-19 patients with RA and $(n=1,035)$ and without $R A(n=137,757)$, the former group had a significantly higher hospitalization rate $(24.7 \%$ vs. $15.6 \%, p<0.05)$ and mortality $(6.3 \%$ vs. $2.5 \%, p<0.05)$. Once hospitalized, the patients with and without RA had similar likelihoods of transferring to the ICU (with RA $18.0 \%$, without RA $16.7 \%, p=0.64$ ) and inpatient mortality (with RA $14.8 \%$, without RA $11.6 \%, p=0.13)$. 
Table 1 Demographics, Prior Medication Use and Selected Outcomes Comparison Between Patients with COVID-19 with Rheumatoid Arthritis and Patients with COVID-19 without Rheumatoid Arthritis

\begin{tabular}{|c|c|c|c|c|}
\hline & $\begin{array}{c}\text { Without RA } \\
\text { pre-matched } \\
(\mathrm{n}=137,757)\end{array}$ & $\begin{array}{c}\text { Without RA } \\
\text { matched } \\
(n=3,042)\end{array}$ & $\begin{array}{l}\text { With RA } \\
(n=1,035)\end{array}$ & $\begin{array}{l}\text { All COVID-19 } \\
(n=138,792)\end{array}$ \\
\hline \multicolumn{5}{|l|}{ Age } \\
\hline Mean (SD) & $45.0(19.7)$ & $61.5(16.6)$ & $61.8(14.6)$ & $45.2(19.7)$ \\
\hline Median [Q1, Q3] & $\begin{array}{l}44.0[29.0 \\
\quad 60.0]\end{array}$ & $\begin{array}{l}64.0[51.0 \\
\quad 73.0]\end{array}$ & $\begin{array}{l}63.0[52.0 \\
72.0]\end{array}$ & $\begin{array}{l}44.0[30.0 \\
\quad 60.0]\end{array}$ \\
\hline Missing & $1,897(1.4 \%)$ & & $15(1.4 \%)$ & $1,912(1.4 \%)$ \\
\hline \multicolumn{5}{|l|}{ Sex } \\
\hline Female & $\begin{array}{l}75,404 \\
(54.7 \%)\end{array}$ & $2446(80.4 \%)$ & $816(78.8 \%)$ & $\begin{array}{l}76,220 \\
(54.9 \%)\end{array}$ \\
\hline Male & $\begin{array}{c}61,432 \\
(44.6 \%)\end{array}$ & $596(19.6 \%)$ & $213(20.6 \%)$ & $\begin{array}{l}61,645 \\
(44.4 \%)\end{array}$ \\
\hline Unknown & $921(0.7 \%)$ & & $6(0.6 \%)$ & $927(0.7 \%)$ \\
\hline \multicolumn{5}{|l|}{ Race } \\
\hline American Indian or Alaska Native & $749(0.5 \%)$ & $17(0.6 \%)$ & $2(0.2 \%)$ & $751(0.5 \%)$ \\
\hline Asian & $3,335(2.4 \%)$ & $44(1.4 \%)$ & $18(1.7 \%)$ & $3,353(2.4 \%)$ \\
\hline Black or African American & $\begin{array}{l}32,089 \\
(23.3 \%)\end{array}$ & $871(28.6 \%)$ & $286(27.6 \%)$ & $\begin{array}{l}32,375 \\
(23.3 \%)\end{array}$ \\
\hline $\begin{array}{l}\text { Native Hawaiian or Other Pacific } \\
\text { Islander }\end{array}$ & $306(0.2 \%)$ & $5(0.2 \%)$ & $0(0 \%)$ & $306(0.2 \%)$ \\
\hline White & $\begin{array}{l}68,925 \\
(50.0 \%)\end{array}$ & $1508(49.6 \%)$ & $528(51.0 \%)$ & $\begin{array}{l}69,453 \\
(50.0 \%)\end{array}$ \\
\hline Unknown & $\begin{array}{l}32,353 \\
(23.5 \%)\end{array}$ & $597(19.6 \%)$ & $201(19.4 \%)$ & $\begin{array}{l}32,554 \\
(23.5 \%)\end{array}$ \\
\hline \multicolumn{5}{|l|}{ Ethnicity } \\
\hline Hispanic or Latino & $\begin{array}{c}25,079 \\
(18.2 \%)\end{array}$ & $398(13.1 \%)$ & $124(12.0 \%)$ & $\begin{array}{l}25,203 \\
(18.2 \%)\end{array}$ \\
\hline Not Hispanic or Latino & $\begin{array}{r}61,425 \\
(44.6 \%)\end{array}$ & $1537(50.5 \%)$ & $541(52.3 \%)$ & $\begin{array}{l}61,966 \\
(44.6 \%)\end{array}$ \\
\hline Unknown & $\begin{array}{r}51,253 \\
(37.2 \%) \\
\end{array}$ & $1107(36.4 \%)$ & $370(35.7 \%)$ & $\begin{array}{c}51,623 \\
(37.2 \%) \\
\end{array}$ \\
\hline \multicolumn{5}{|l|}{ Smoking } \\
\hline No & $\begin{array}{l}128,754 \\
(93.5 \%)\end{array}$ & $2320(76.3 \%)$ & $781(75.5 \%)$ & $\begin{array}{l}129,535 \\
(93.3 \%)\end{array}$ \\
\hline Yes & $9,003(6.5 \%)$ & $722(23.7 \%)$ & $254(24.5 \%)$ & $9257(6.7 \%)$ \\
\hline \multicolumn{5}{|l|}{ BMI (kg/m2) } \\
\hline Mean (SD) & $27.8(5.94)$ & $28.5(5.93)$ & $28.8(6.02)$ & $27.8(5.94)$ \\
\hline Median [Q1, Q3] & $\begin{array}{c}27.0 \\
{[23.0,32.0]}\end{array}$ & $\begin{array}{c}28.0 \\
{[24.0,33.0]}\end{array}$ & $\begin{array}{c}29.0 \\
{[25.0,33.0]}\end{array}$ & $\begin{array}{c}27.0 \\
{[23.0,32.0]}\end{array}$ \\
\hline Missing & $\begin{array}{l}97,928 \\
(71.1 \%)\end{array}$ & $2002(65.8 \%)$ & $490(47.3 \%)$ & $\begin{array}{l}98,418 \\
(70.9 \%)\end{array}$ \\
\hline $\mathrm{CCl}$ (weighted score based on & & & & \\
\hline
\end{tabular}


medRxiv preprint doi: https://doi.org/10.1101/2021.07.09.21260106; this version posted July 12, 2021. The copyright holder for this preprint (which was not certified by peer review) is the author/funder, who has granted medRxiv a license to display the preprint in perpetuity.

All rights reserved. No reuse allowed without permission.

\begin{tabular}{|c|c|c|c|c|}
\hline comorbid conditions) & & & & \\
\hline Mean (SD) & $0.660(1.68)$ & $1.39(2.39)$ & $3.07(2.86)$ & $0.678(1.71)$ \\
\hline Median [Q1, Q3] & $0.00[0.00$, & $0[0,2.00]$ & $2.00[1.00$, & $0.00[0.00$, \\
& $0.00]$ & & $4.50]$ & $0.00]$ \\
\hline
\end{tabular}

bDMARD = biologics disease-modifying antirheumatic drugs; $\mathrm{CCl}=$ Charlson Comorbidity Index; COVID-19= Coronavirus disease 2019; csDMARD = conventional synthetic disease-modifying antirheumatic drugs; ICU = intensive care unit; RA = Rheumatoid Arthritis; SD = standard deviation; tsDMARD = targeted synthetic disease-modifying antirheumatic drugs; Q1 = quartile 1; Q3 = quartile 3.

a Only among hospitalized patients. 
medRxiv preprint doi: https://doi.org/10.1101/2021.07.09.21260106; this version posted July 12, 2021. The copyright holder for this preprint (which was not certified by peer review) is the author/funder, who has granted medRxiv a license to display the preprint in perpetuity. All rights reserved. No reuse allowed without permission.

Table 1 Demographics, Prior Medication Use and Selected Outcomes Comparison Between Patients with COVID-19 with Rheumatoid Arthritis and Patients with COVID-19 without Rheumatoid Arthritis (cont.)

\begin{tabular}{|c|c|c|c|c|}
\hline & $\begin{array}{l}\text { Without RA } \\
(n=137,757)\end{array}$ & $\begin{array}{c}\text { Without RA } \\
\text { matched } \\
(n=3,042)\end{array}$ & $\begin{array}{l}\text { With RA } \\
(n=1,035)\end{array}$ & $\begin{array}{l}\text { All COVID-19 } \\
(n=138,792)\end{array}$ \\
\hline \multicolumn{5}{|c|}{ Steroids } \\
\hline No & $\begin{array}{l}116,819 \\
(84.8 \%)\end{array}$ & $2349(77.2 \%)$ & $497(48.0 \%)$ & $\begin{array}{l}117,316 \\
(84.5 \%)\end{array}$ \\
\hline Yes & $\begin{array}{c}20,938 \\
(15.2 \%) \\
\end{array}$ & $693(22.8 \%)$ & $538(52.0 \%)$ & $\begin{array}{l}21,476 \\
(15.5 \%) \\
\end{array}$ \\
\hline \multicolumn{5}{|c|}{ Anti-coagulants } \\
\hline No & $\begin{array}{l}134,593 \\
(97.7 \%)\end{array}$ & $2888(94.9 \%)$ & $945(91.3 \%)$ & $\begin{array}{l}135,538 \\
(97.7 \%)\end{array}$ \\
\hline Yes & $3,164(2.3 \%)$ & $154(5.1 \%)$ & $90(8.7 \%)$ & $3,254(2.3 \%)$ \\
\hline \multicolumn{5}{|c|}{ Any DMARD } \\
\hline No & $\begin{array}{l}136286 \\
(98.9 \%)\end{array}$ & $2985(98.1 \%)$ & $601(58.1 \%)$ & $\begin{array}{l}136887 \\
(98.6 \%)\end{array}$ \\
\hline Yes & $1471(1.1 \%)$ & 57 (1.9\%) & $434(41.9 \%)$ & 1905 (1.4\%) \\
\hline \multicolumn{5}{|c|}{ csDMARD } \\
\hline No & $\begin{array}{l}136,722 \\
(99.2 \%)\end{array}$ & 2997 (98.5\%) & $699(67.5 \%)$ & $\begin{array}{l}137,421 \\
(99.0 \%)\end{array}$ \\
\hline Yes & $1,035(0.8 \%)$ & $45(1.5 \%)$ & $336(32.5 \%)$ & $1,371(1.0 \%)$ \\
\hline \multicolumn{5}{|c|}{ tsDMARD } \\
\hline No & $\begin{array}{c}137,705 \\
(100.0 \%)\end{array}$ & $3042(100 \%)$ & 994 (96.0\%) & $\begin{array}{l}138,699 \\
(99.9 \%)\end{array}$ \\
\hline Yes & $52(0.0 \%)$ & $0(0 \%)$ & $41(4.0 \%)$ & $93(<0.1 \%)$ \\
\hline \multicolumn{5}{|c|}{ bDMARD } \\
\hline No & $\begin{array}{l}137,265 \\
(99.6 \%)\end{array}$ & 3024 (99.4\%) & $844(81.5 \%)$ & $\begin{array}{l}138,109 \\
(99.5 \%)\end{array}$ \\
\hline Yes & $492(0.4 \%)$ & $18(0.6 \%)$ & $191(18.5 \%)$ & $683(0.5 \%)$ \\
\hline \multicolumn{5}{|c|}{ Hospitalization } \\
\hline No & $\begin{array}{l}116,322 \\
(84.4 \%)\end{array}$ & $2342(77.0 \%)$ & $779(75.3 \%)$ & $\begin{array}{l}117,101 \\
(84.4 \%)\end{array}$ \\
\hline Yes & $\begin{array}{c}21,435 \\
(15.6 \%) \\
\end{array}$ & $700(23.0 \%)$ & $256(24.7 \%)$ & $\begin{array}{c}21,691 \\
(15.6 \%)\end{array}$ \\
\hline \multicolumn{5}{|l|}{$\mathrm{ICU}^{\mathrm{a}}$} \\
\hline No & $\begin{array}{c}17,861 \\
(83.3 \%)\end{array}$ & $605(81.2 \%)$ & $210(82.0 \%)$ & $\begin{array}{c}18,071 \\
(83.3 \%)\end{array}$ \\
\hline Yes & $\begin{array}{c}3,574 \\
(16.7 \%)\end{array}$ & $140(18.8 \%)$ & $46(18.0 \%)$ & $\begin{array}{c}3,620 \\
(16.7 \%)\end{array}$ \\
\hline \multicolumn{5}{|c|}{ Inpatient mortality $^{a}$} \\
\hline No & $\begin{array}{c}18,949 \\
(88.4 \%)\end{array}$ & $638(85.6 \%)$ & $218(85.2 \%)$ & $\begin{array}{c}19,167 \\
(88.4 \%)\end{array}$ \\
\hline
\end{tabular}


medRxiv preprint doi: https://doi.org/10.1101/2021.07.09.21260106; this version posted July 12, 2021. The copyright holder for this preprint (which was not certified by peer review) is the author/funder, who has granted medRxiv a license to display the preprint in perpetuity. All rights reserved. No reuse allowed without permission.

\begin{tabular}{|l|c|c|c|c|}
\hline Yes & $\begin{array}{c}2,486 \\
(11.6 \%)\end{array}$ & $107(14.4 \%)$ & $38(14.8 \%)$ & $\begin{array}{c}2,524 \\
(11.6 \%)\end{array}$ \\
\hline Overall mortality & & & & \\
\hline No & $\begin{array}{c}134,249 \\
(97.5 \%)\end{array}$ & $\begin{array}{c}2,879 \\
(94.6 \%)\end{array}$ & $970(93.7 \%)$ & 135,219 \\
& $3,508(2.5 \%)$ & $163(5.4 \%)$ & $65(6.3 \%)$ & $3,573(2.6 \%)$ \\
\hline Yes & $3 \%)$ & \\
\hline bDMARD = biologics disease-modifying antirheumatic drugs; CCI = Charlson Comorbidity Index; \\
COVID-19= Coronavirus disease 2019; csDMARD = conventional synthetic disease-modifying \\
antirheumatic drugs; ICU = intensive care unit; RA = Rheumatoid Arthritis; SD = standard deviation; \\
tsDMARD = targeted synthetic disease-modifying antirheumatic drugs; Q1 = quartile 1; Q3 = quartile 3. \\
a Only among hospitalized patients. \\
\hline
\end{tabular}


medRxiv preprint doi: https://doi.org/10.1101/2021.07.09.21260106; this version posted July 12, 2021. The copyright holder for this preprint (which was not certified by peer review) is the author/funder, who has granted medRxiv a license to display the preprint in perpetuity.

All rights reserved. No reuse allowed without permission.

We matched 3,042 non-RA COVID-19 patients to 1,014 RA COVID-19 patients and 745 hospitalized non-RA COVID-19 patients to 249 hospitalized RA COVID-19 patients. All matching variables were well balanced post-match (Figure S1/S2). When comparing non-hospitalized patients with RA and matched patients without RA, we found no significant difference in the odds of hospitalization and mortality (hospitalization odds ratio [OR] 1.08, 95\% confidence interval [Cl]: [0.88,1.33]), mortality (OR 0.93, 95\% [Cl]: [0.65,1.34]). For matched hospitalized COVID-19 patients with RA $(n=249)$ and patients without RA $(n=745)$, we did not find significant differences in odds of transferring to ICU, or death during hospitalization (ICU: OR 0.94, 95\% [Cl]: [0.60,1.45], inpatient mortality: OR 0.86, 95\% [Cl]: [0.53,1.40]) (see Table 2). Treatments up to a year prior to the index date were also evaluated (Table 3). We found no significant effects except for anti-coagulants on the risk of hospitalization (OR 1.49, 95\% [Cl]: $[1.10,2.02])$ and overall mortality (OR 1.62, 95\% [CI]: [1.04, 2.51]).

Our sensitivity analyses looking at laboratory-confirmed COVID-19 cases reduced our sample size by $32 \%$. Results remained unchanged except that confidence intervals were wider (table S4-S6).

Table 2 Outcomes Among Patients with Rheumatoid Arthritis with COVID-19 and Matched Controls

\begin{tabular}{|c|c|c|c|c|c|}
\hline \multicolumn{6}{|c|}{ a. Outcomes all COVID-19 patients } \\
\hline & $\begin{array}{c}\text { Matched } \\
\text { Without RA } \\
(n=3042)\end{array}$ & $\begin{array}{l}\text { With RA } \\
(n=1014)\end{array}$ & $\begin{array}{c}\text { Overall } \\
(n=4056)\end{array}$ & $\begin{array}{l}\text { Crude OR } \\
(95 \% \mathrm{Cl})\end{array}$ & $\begin{array}{l}\text { Adjusted OR } \\
\quad(95 \% \mathrm{Cl})\end{array}$ \\
\hline \multicolumn{6}{|c|}{ Hospitalization } \\
\hline No & $\begin{array}{c}2,342 \\
(77.0 \%)\end{array}$ & $\begin{array}{c}765 \\
(75.4 \%)\end{array}$ & $\begin{array}{c}3,107 \\
(76.6 \%)\end{array}$ & $\begin{array}{c}1.78 \\
(1.55,2.06)\end{array}$ & $\begin{array}{c}1.08 \\
(0.88,1.33)\end{array}$ \\
\hline Yes & $\begin{array}{c}700 \\
(23.0 \%)\end{array}$ & $\begin{array}{c}249 \\
(24.6 \%)\end{array}$ & $\begin{array}{c}949 \\
(23.4 \%)\end{array}$ & & \\
\hline \multicolumn{6}{|c|}{ Mortality } \\
\hline No & $\begin{array}{c}2,879 \\
(94.6 \%)\end{array}$ & $\begin{array}{c}953 \\
(94.0 \%)\end{array}$ & $\begin{array}{c}3,832 \\
(94.5 \%)\end{array}$ & $\begin{array}{c}2.56 \\
(1.99,3.30)\end{array}$ & $\begin{array}{c}0.93 \\
(0.65,1.34)\end{array}$ \\
\hline Yes & $163(5.4 \%)$ & $\begin{array}{c}61 \\
(6.0 \%)\end{array}$ & $\begin{array}{c}224 \\
(5.5 \%)\end{array}$ & & \\
\hline \multicolumn{6}{|c|}{ b. Outcomes among hospitalized COVID-19 patients } \\
\hline & $\begin{array}{c}\text { Matched } \\
\text { Without RA } \\
(n=745)\end{array}$ & $\begin{array}{l}\text { With RA } \\
(n=249)\end{array}$ & $\begin{array}{l}\text { Overall } \\
(n=994)\end{array}$ & $\begin{array}{l}\text { Crude OR } \\
(95 \% \mathrm{Cl})\end{array}$ & $\begin{array}{l}\text { Adjusted OR }{ }^{a} \\
\quad(95 \% \mathrm{Cl})\end{array}$ \\
\hline \multicolumn{6}{|l|}{ ICU } \\
\hline No & $\begin{array}{c}605 \\
(81.2 \%)\end{array}$ & $\begin{array}{c}204 \\
(81.9 \%)\end{array}$ & $\begin{array}{c}809 \\
(81.4 \%)\end{array}$ & $\begin{array}{c}1.09 \\
(0.791 .51)\end{array}$ & $\begin{array}{c}0.94 \\
(0.60,1.45)\end{array}$ \\
\hline Yes & $\begin{array}{c}140 \\
(18.8 \%)\end{array}$ & $\begin{array}{c}45 \\
(18.1 \%)\end{array}$ & $\begin{array}{c}185 \\
(18.6 \%)\end{array}$ & & \\
\hline Inpatie & & & & & \\
\hline
\end{tabular}


medRxiv preprint doi: https://doi.org/10.1101/2021.07.09.21260106; this version posted July 12, 2021. The copyright holder for this preprint (which was not certified by peer review) is the author/funder, who has granted medRxiv a license to display the preprint in perpetuity.

\begin{tabular}{|c|c|c|c|c|c|} 
mortality & & & & \\
\hline No & $\begin{array}{c}638 \\
(85.6 \%)\end{array}$ & $\begin{array}{c}213 \\
(85.5 \%)\end{array}$ & $\begin{array}{c}851 \\
(85.6 \%)\end{array}$ & $\begin{array}{c}1.33 \\
(0.94,1.88)\end{array}$ & $\begin{array}{c}0.86 \\
(0.53,1.40)\end{array}$ \\
\hline Yes & 107 & 36 & 143 & & \\
\hline
\end{tabular}

$\mathrm{Cl}=$ confidence interval; $\mathrm{OR}=$ odds ratio; $\mathrm{RA}=\mathrm{Rheumatoid}$ Arthritis.

${ }^{\text {a }}$ Results are based on multiple logistic regression adjusting for age, race/ethnicity, sex, body mass index (BMI), comorbidities, and pre-index medication such as steroids, anti-coagulants, antibiotics, cSDMARD and bDMARD.

Table 3 The effects of treatment on different outcomes evaluated

\begin{tabular}{|c|c|c|c|c|}
\hline \multirow[b]{2}{*}{ Treatment } & \multicolumn{2}{|c|}{$\begin{array}{l}\text { Outcomes among all COVID-19 } \\
\text { patients } \\
\qquad \text { OR }(95 \% \mathrm{Cl})\end{array}$} & \multicolumn{2}{|c|}{$\begin{array}{l}\text { Outcomes among all Hospitalized } \\
\text { COVID-19 patients } \\
\text { OR }(95 \% \mathrm{Cl})\end{array}$} \\
\hline & Hospitalization & os & ICU & Inpatient mortality \\
\hline Steroids & $0.83(0.68,1.01)$ & $0.99(0.70,1.40)$ & $0.93(0.60,1.44)$ & $0.85(0.53,1.37)$ \\
\hline Anti-coagulants & $1.49(1.10,2.02)$ & $1.62(1.04,2.51)$ & $1.68(0.97,2.94)$ & $1.44(0.78,2.68)$ \\
\hline csDMARD & $0.76(0.55,1.04)$ & $0.66(0.36,1.22)$ & $0.73(0.35,1.52)$ & $0.76(0.33,1.76)$ \\
\hline bDMARD & $0.69(0.45,1.05)$ & $1.19(0.58,2.42)$ & $1.87(0.78,4.49)$ & $2.42(0.93,6.29)$ \\
\hline \multicolumn{5}{|c|}{$\begin{array}{l}\mathrm{Cl}=\text { confidence interval; } \mathrm{OR}=\text { odds ratio; } \mathrm{RA}=\mathrm{Rheumatoid} \text { Arthritis; } \mathrm{OS}=\text { overall survival. } \\
\text { Results are based on multiple logistic regression adjusting for sex, age, race/ethnicity, body } \\
\text { mass index (BMI), and comorbidities. }\end{array}$} \\
\hline
\end{tabular}


medRxiv preprint doi: https://doi.org/10.1101/2021.07.09.21260106; this version posted July 12, 2021. The copyright holder for this preprint

(which was not certified by peer review) is the author/funder, who has granted medRxiv a license to display the preprint in perpetuity.

All rights reserved. No reuse allowed without permission.

\section{Discussion}

It has been hypothesized that persons using immunosuppressive medications might be at higher risk of severe COVID-19 outcomes due to a compromised immune system and inability to suppress early viral replication. However, we did not find evidence to suggest patients with COVID-19 and RA are more likely to have severe outcomes after matching. We also did not find any effect of pre-COVID-19 DMARD treatments on the risk of COVID-19 related outcomes.

There is a growing body of conflicting literature examining COVID-19 outcomes among RA patients. While some studies reported increased risk of hospitalization ${ }^{15}$ and death ${ }^{16}$ among combined populations of patients with inflammatory conditions, specific analyses focusing on RA populations have suggested that there is no increased risk of hospitalization. ${ }^{77}$ However, when examining RA treatments, some studies suggest a possible protective effect of b/tsDMARDs ${ }^{7}$ and anti-TNF therapy ${ }^{715}$ on risk of hospitalization, while increased risk of hospitalization was reported for JAK inhibitor users ${ }^{15}$. Directionally, findings from our paper also suggested similar results, with a non-statistically significant reduced risk of hospitalization among bDMARD users. Subsequent analysis of the hospitalized patients showed nonstatistically significant elevated risks of ICU stay and mortality. Notably, in our sensitivity analyses using only lab confirmed COVID-19 cases, the directional association showing reduced risk of hospitalization remained for all COVID-19 patients, while among hospitalized patients, the risk of ICU and mortality was no longer elevated.

When characterizing patients with and without severe outcomes, we found older age, male gender, smokers, and higher $\mathrm{CCl}$ are associated with severe outcomes (online supplemental table S7-S8). Patients utilizing anti-coagulants in the pre-COVID-19 period had significantly increased risk of hospitalization and mortality (Table 3). Similar, non-significant results were found among hospitalized patients (online supplemental table S9-S10). The effect of anticoagulant use was the same across populations with or without RA.

COVID-19 is known to be associated with abnormal coagulation, specifically higher clot formation ${ }^{12}$ and subsequent increased risk of mortality. ${ }^{13}{ }^{14}$ Patients using anti-coagulants may have comorbidities that predispose them to worse outcomes from COVID-19 (cardiovascular disease, malignancy, or another hypercoagulable state) despite the potential protective effect of the anti-coagulant itself. Alternatively, the anti-coagulants themselves could paradoxically be causing worse COVID-19 outcomes, perhaps by increasing the risk of bleeding complications. Distinguishing between these hypotheses could have important implications for the treatment of patients with COVID-19; however, further study is required.

Although the scope of this study is limited to information captured by participating HCOs and might not be reflective of patients' complete medical encounters, medication use and detailed disease conditions such as disease activities, this is the largest published study examining the risk of severe outcomes among patients with COVID-19 and RA to date. Worse COVID-19 outcomes were not seen among patients with RA, regardless of historical RA therapy. These findings suggest that asymptomatic patients with RA should stay on their routine RA therapy, though it is still unclear whether or not RA or other medications should be discontinued after COVID-19 infection. 
medRxiv preprint doi: https://doi.org/10.1101/2021.07.09.21260106; this version posted July 12, 2021. The copyright holder for this preprint (which was not certified by peer review) is the author/funder, who has granted medRxiv a license to display the preprint in perpetuity.

All rights reserved. No reuse allowed without permission.

\section{References}

1. Dong E, Du H, Gardner L. An interactive web-based dashboard to track COVID-19 in real time. Lancet Infect Dis2020;20:533-34 https://doi.org/10.1016/S1473-3099(20)30120-1.

2. Incerti D, Rizzo S, Li X, et al. Risk factors for mortality among hospitalized patients with COVID-19 medRxivPublished Online First: 2 October 2020.

https://doi.org/10.1101/2020.09.22.20196204

3. Mueller AL, McNamara MS, Sinclair DA. Why does COVID-19 disproportionately affect older people?. Aging (Albany NY)202012;10:9959-81

https://doi.org/10.18632/aging.103344.

4. Sanyaolu A, Okorie C, Marinkovic A, et al. Comorbidity and its impact on patients with COVID-19. SN Compr Clin Med2020;1-8 https://doi.org/10.1007/s42399-020-00363-4.

5. Tartof SY, Qian L, Hong V, et al. Obesity and mortality among patients diagnosed with COVID-19: results from an integrated health care organization. Ann Intern Med2020;173:773-81 https://doi.org/10.7326/M20-3742.

6. Michaud K, Wipfler K, Shaw Y, et al. Experiences of patients with rheumatic diseases in the united states during early days of the COVID-19 pandemic. ACR Open Rheumatol 2020;2:335-43 https://doi.org/10.1002/acr2.11148.

7. Gianfrancesco M, Hyrich $\mathrm{KL}, \mathrm{Al}$-Adely $\mathrm{S}$, et al. Characteristics associated with hospitalisation for COVID-19 in people with rheumatic disease: data from the COVID-19 global rheumatology alliance physician-reported registry. Ann Rheum Dis2020;79:859-66 https://doi.org/10.1136/annrheumdis-2020-217871.

8. Richez C, Flipo R, Berenbaum F, et al. Managing patients with rheumatic diseases during the COVID-19 pandemic: the french society of rheumatology answers to most frequently asked questions up to may 2020 Joint Bone Spine2020;87:431-37 https://doi.org/10.1016/j.jbspin.2020.05.006.

9. Georgiev T. Coronavirus disease 2019 (COVID-19) and anti-rheumatic drugs. Rheumatol Int2020;40:825-26 https://doi.org/10.1007/s00296-020-04570-z.

10. Monti S, Balduzzi S, Delvino P, et al. Clinical course of COVID-19 in a series of patients with chronic arthritis treated with immunosuppressive targeted therapies. Ann Rheum Dis2020;79:667-68 https://doi.org/10.1136/annrheumdis-2020-217424.

11. Favalli EG, Monti S, Ingegnoli F, et al. Incidence of COVID-19 in patients with rheumatic diseases treated with targeted immunosuppressive drugs: what can we learn from observational data?. Arthritis Rheumato/2020;72:1600-06 https://doi.org/10.1002/art.41388.

12. Magro C, Mulvey JJ, Berlin D, et al. Complement associated microvascular injury and thrombosis in the pathogenesis of severe COVID-19 infection: a report of five cases. Transl Res2020;220:1-13 https://doi.org/10.1016/j.trsl.2020.04.007. 
medRxiv preprint doi: https://doi.org/10.1101/2021.07.09.21260106; this version posted July 12, 2021. The copyright holder for this preprint

(which was not certified by peer review) is the author/funder, who has granted medRxiv a license to display the preprint in perpetuity.

All rights reserved. No reuse allowed without permission.

13. Connors JM., Levy JH. COVID-19 and its implications for thrombosis and anticoagulation. Blood2020;135:2033-40 https://doi.org/10.1182/blood.2020006000.

14. Al-Samkari $\mathrm{H}$, Karp Leaf RS, Dzik WH, et al. COVID-19 and coagulation: bleeding and thrombotic manifestations of SARS-CoV-2 infection. Blood2020;136:489-500 https://doi.org/10.1182/blood.2020006520.

15. Haberman, Rebecca H., Rochelle Castillo, Alan Chen, Di Yan, Deborah Ramirez, Vaish Sekar, Robert Lesser, et al. n.d. "COVID-19 in Patients with Inflammatory Arthritis: A Prospective Study on the Effects of Comorbidities and DMARDs on Clinical Outcomes." Arthritis \& Rheumatology n/a (n/a). Accessed August 14, 2020.

https://doi.org/10.1002/art.41456.

16. Williamson, Elizabeth J., Alex J. Walker, Krishnan Bhaskaran, Seb Bacon, Chris Bates, Caroline E. Morton, Helen J. Curtis, et al. 2020. "Factors Associated with COVID-19Related Death Using OpenSAFELY." Nature 584 (7821): 430-36. https://doi.org/10.1038/s41586-020-2521-4.

17. Hyrich, Kimme L., and Pedro M. Machado. 2020. "Rheumatic Disease and COVID-19: Epidemiology and Outcomes." Nature Reviews. Rheumatology, December, 1-2. https://doi.org/10.1038/s41584-020-00562-2. 